\title{
Optimization of Modular Wiring Harnesses by Means of Regression Models for Temperature Prediction of Wire Bundles
}

\author{
A. Rius, A. Garcia and M.A. Díaz
}

\begin{abstract}
Automotive wiring harnesses have become heavier and more complex due to their increasing number of electrical components. It is now desired to reduce their mass of copper. For this purpose, experimentation can be partially replaced by simulation, but it is still impossible to exhaustively simulate all of the combinations of modular wiring harness. This proposed approach consists of carrying out simulations using the FEM method and using their results to create regression models. Polynomial formulae can give the same information as simulations within a clearly reduced time and satisfying accuracy. An optimization algorithm introduced in this study will use them to assign new cable cross-sections of harnesses considering their currents and the ambient temperature.
\end{abstract}

Keywords Wire harness - Optimization - Weight reduction - Regression • Models · Temperature prediction

\section{Introduction}

The number and overall weight of electric components commercial vehicles has risen across the recent decades. Some European Automotive brands offer wide possibilities of customization for their vehicles, so that customers can choose features of their cars among a large variety. Furthermore, worldwide car sales demand

\footnotetext{
A. Rius $(\bowtie) \cdot$ A. Garcia

Universitat Politècnica de Catalunya (UPC), Barcelona, Spain

e-mail: extern.armand.rius@seat.es; armand.rius.rueda@gmail.com
}
A. Garcia
e-mail: garciae@ee.upc.edu

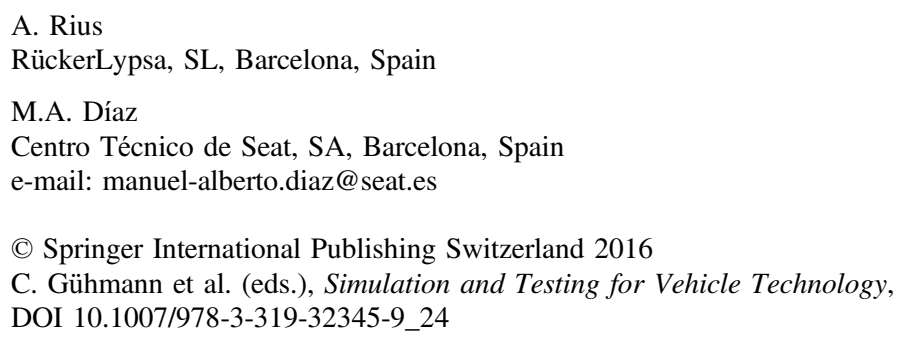


extra customization capabilities for car manufactures to offer specific features of their models adapted for each continent. In terms of magnitude, the overall number of possible combinations of features of a car model can reach $10^{10}$.

Different simulators have been developed to predict the temperature of wires or wire bundles in vehicle applications, with the aim of optimization. Nevertheless, the huge number of combinations makes it unaffordable to simulate all of the feature combinations due to a matter of computation time.

Wires must be dimensioned in accordance to their worst case. This involves the fact that wires in bundles transmit heat amongst them. Therefore, a wire must be dimensioned observing other wires in all of the segments of the wiring harness throughout it extends.

The task of optimizing the mass of copper maintaining high reliability in such complex systems demands extremely fast ways of predicting temperature. This document proposes using regression models with the form of multidimensional polynomials providing the necessary information to carry out this task.

\subsection{Scope}

Wiring harnesses are generally assembled manually, so that all of its segments contain sets of undistributed wires forming random layouts. Thus, it is mandatory to design wire harnesses in such a way that the internal distribution of the bundles do not affect their maximum temperature. This latter requirement demands that all bundles have homogeneity in their heat generation, viewed as the $f$ parameter $\left(\mathrm{W} / \mathrm{m}^{3}\right)$ in the heat dissipation equation, which must be roughly uniform all over the cross-section of any random bundle. It must be assumed that $f$ equals to zero in all regions of a bundle except in conductor regions (generally copper), whose Joule effect heat depends on their current density $J$ and resistivity $\rho$ responds to the following equation:

$$
f=J^{2} \cdot \rho(T)
$$

The latter equation presents the resistivity of copper as a function of the temperature, $T$. Since the resistivity $\rho$ is related to the conductor material, if we want to keep $f$ uniform over a cross-section of a bundle containing wires with different conductor materials, $J$ will have to be adapted to each of them so that $f$ is approximately uniform. The different dependency of $\rho$ on the temperature for different materials complicates this, but it is still possible to find all the values of $J$ assuming a given temperature, which could be a certain point of temperature between the ambient temperature and the maximum expected temperature of the bundle. This document only explores the case of bundles with unique composition of materials (copper and polyvinylchloride).

Nonetheless, power wire bundles will frequently include signal wires carrying negligible currents, which prevent us to maintain this desired unique Joule heat per unit volume $f$ for all of the wires. These signal wires will have nearly zero heat 
dissipation in their cores, since the wire manufacturers cannot provide wires with unlimitedly thin diameters. No matter how low the current might be, these wires will always be cold, i.e. providing nearly $f=0$ heat to the system. Their presence enlarges de bundle and provides negligible Joule heat at the same time. The resulting bundle dissipates slightly more heat by means of convection and radiation, due to a greater surface of contact with the environment, when compared with the same bundle lacking these signal wires.

These aspects make us understand that these cold wires can only benefit bundles by lowering their maximum expectable temperature for given currents. Since wire dimensioning must be done according to a reasonable worst case, this document deals solely with bundles containing just power wires with approximately uniform current density, $J$.

\subsection{Critical Dimensions of a Homogeneous Conductive Cylinder with Uniform Current Density}

If all of the wires in the bundle are made of the same materials and they have the same current density, the temperature of the center will increase with the radius of the bundle. Therefore, it is possible to find a certain critical area in the bundle $A_{\text {crit }}$ corresponding to the area that makes the temperature of the bundle reach certain maximum acceptable temperature $T_{\max }$ for a given current density, $J$. Assuming for a moment that the bundle is a cylinder of conductive material with a uniform current density $J$, the heat equation in steady state might be written as follows:

$$
-\lambda \cdot 2 \pi r L \cdot \frac{d T}{d r}=f \cdot \pi r^{2} L=F
$$

where $\lambda$ is the thermal conductivity of the cylinder material, and $r$ and $L$ are respectively its radius and its length. Integrating from $r=0$ where the maximum temperature $T_{c}$ is found, up to $r=R$, where the temperature is $T_{s}$ :

$$
T_{c}-T_{s}=\frac{f R^{2}}{4 \lambda}=\frac{f A}{4 \pi \lambda}
$$

Now, the temperature of the external surface of the cylinder can be found by equaling the entire generated heat $F$ with all the dissipated heat due to radiation and convection:

$$
\begin{gathered}
2 \pi R L \cdot \alpha\left(T_{s}\right) \cdot\left(T_{s}-T_{a}\right)=\pi R^{2} L f=F \\
\rightarrow T_{s}=\frac{f R}{2 \tilde{\alpha}}+T_{a}
\end{gathered}
$$


Fig. 1 Center temperature in a cylinder of cross-section $A$ and uniform heat generation $f$

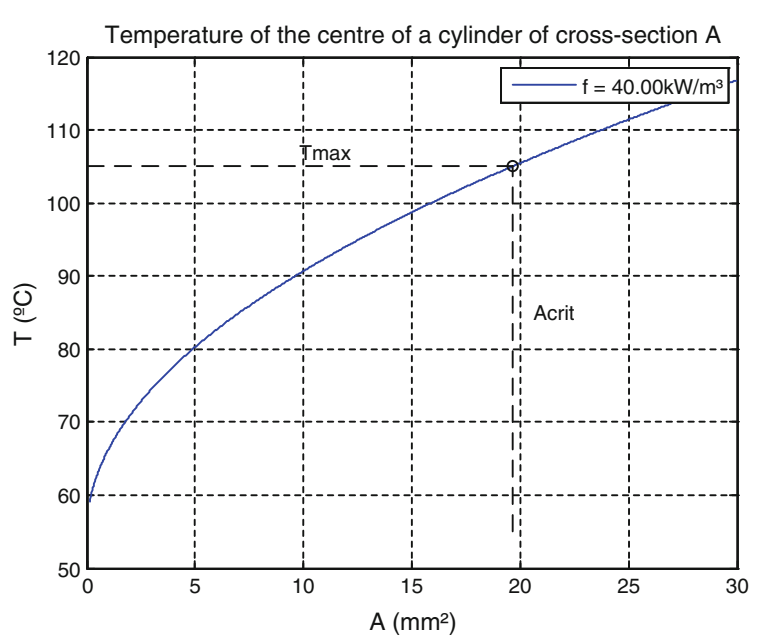

Here, an averaged and constant value of $\alpha(T)\left(\mathrm{W} / \mathrm{m}^{2} \mathrm{~K}\right)$ is used. Now, the maximum temperature in the center and its dependence with the cross-section of the cylinder can be found:

$$
\begin{gathered}
T_{c}=T_{a}+\frac{f R^{2}}{4 \lambda}+\frac{f R}{2 \tilde{\alpha}} \\
T_{c}=f\left(\frac{A}{4 \pi \lambda}+\frac{\sqrt{A}}{2 \tilde{\alpha} \sqrt{\pi}}\right)+T_{a}
\end{gathered}
$$

Equation (1) is represented graphically in Fig. 1. Equaling $T_{c}$ to a maximum value of temperature $T_{\text {max }}$, the relation between $f$ and its critical radius $R_{\max }$ can be found, and this way $A_{\max } \equiv A_{\text {crit }}$ is obtained:

$$
\begin{gathered}
R_{\max }=-\frac{\lambda}{\tilde{\alpha}}+\sqrt{\frac{\lambda^{2}}{\tilde{\alpha}^{2}}+\frac{4 \lambda\left(T_{0}-T_{a m b}\right)}{f}} \\
A_{\max }=4 \pi \lambda^{2}\left(-\frac{1}{2 \tilde{\alpha}}+\sqrt{\frac{1}{4 \tilde{\alpha}^{2}}+\frac{T_{0}-T_{a m b}}{\lambda J^{2} \rho}}\right)^{2}
\end{gathered}
$$

This can be seen graphically in Fig. 2 .

Conversely, it is useful to consider the minimum area $A_{\min }$ as a function of the total current $I$ rather than the current density, Fig. 3.

It is also convenient to consider the maximum current $I_{\max }$ as a function of the total cross-sectional area of the cylinder, Fig. 4. 
Fig. 2 Maximum area of a cylinder conductor set to uniform current density $J$

Fig. 3 Minimum cross-sectional area of a cylindrical conductor subjected to a current $I$ and uniform current density. Smaller areas would produce temperatures above $T_{\max }$

Fig. 4 Maximum current for a cylindrical conductor with cross-sectional area $A$. Greater currents would produce temperatures above the maximum acceptable value
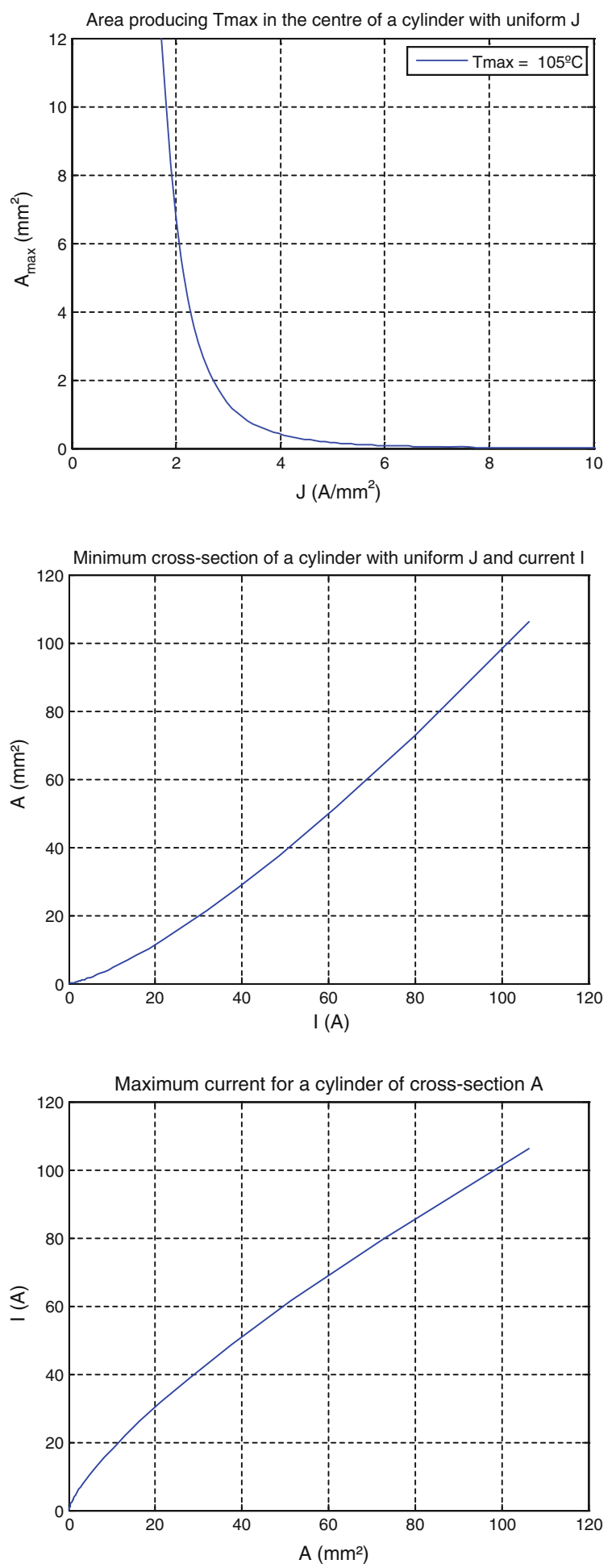
These latter relations are interesting, since they allow for estimating the maximum area of an arbitrary bundle, given the sum of its currents or its area. This will be used later on in this document.

\section{Critical Currents of Wire Bundles Found by FEM Simulations}

Actual wire bundles can be regarded as a heterogeneous mix of different materials, which are reduced here to copper, still dry air, polyvinylchloride (PVC) and poly-ethylene terephthalate (PET) fleece. The behavior of this system is similar to the case of the prior plotted homogeneous cylinder, yet not as simple to express by means of its parameters, such as $\lambda$ or $\rho$.

In order to observe the corresponding curves for actual heterogeneous bundles, finite-element simulations have been carried out. In order to allow radial symmetry, simulated bundles contain specific amounts of wires forming radial symmetry around a central wire $(N=6 n+1=1,7,13,19,25,31,37 \ldots)$, all of them having the same diameter. This ensures all bundles have approximately round shapes. Round shapes with uniform current density are capable of producing the worst-case hot spot in the center of the bundle, and they are the typical shape of manufactured segments of wiring harnesses.

The critical value of current density of each bundle is found by means of iterative simulations, which try to raise the temperature at the center to exactly $T_{\max }=105^{\circ} \mathrm{C}$, which is one of the standard values of acceptable steady temperature for PVC in vehicle wiring applications.

Founding the critical current $I_{\text {crit }}$ of the bundle for a given area instead of the opposite has the advantage that the geometry and the mesh of the finite elements are generated only once and reused for each new iterated simulation, which speeds up the process. The resulting list of area-current pairs can be swapped afterwards to either obtain the maximum current for a given area or the minimum area for a given current.

We have maintained an ambient temperature of $70{ }^{\circ} \mathrm{C}$ for the first set of simulations in order to explore the possibilities of these hypotheses. The $\mathrm{x}$-axis is the sum of all the cross-section areas of the wires - areas of their conductors-Fig. 5.

We can observe a well-defined curve with the same characteristics as the one of the homogeneous cylinder, Fig. 5. Again, we plot the critical area of the bundle (the sum of all of the copper areas) versus the sum of all of the currents, and we can fit a third degree polynomial to it, which provides tight prediction bounds, Fig. 6.

This polynomial expression plotted in Fig. 6 can be used to estimate the critical area of a bundle, given its sum of currents. Wires might be dimensioned assigning areas so that the sum of all of the areas of copper equals this value of critical area. The fact that available cross-sections in industry are discrete values turns this one 
Fig. 5 Results of critical current densities of wire bundles versus their areas of copper obtained by FEM simulations

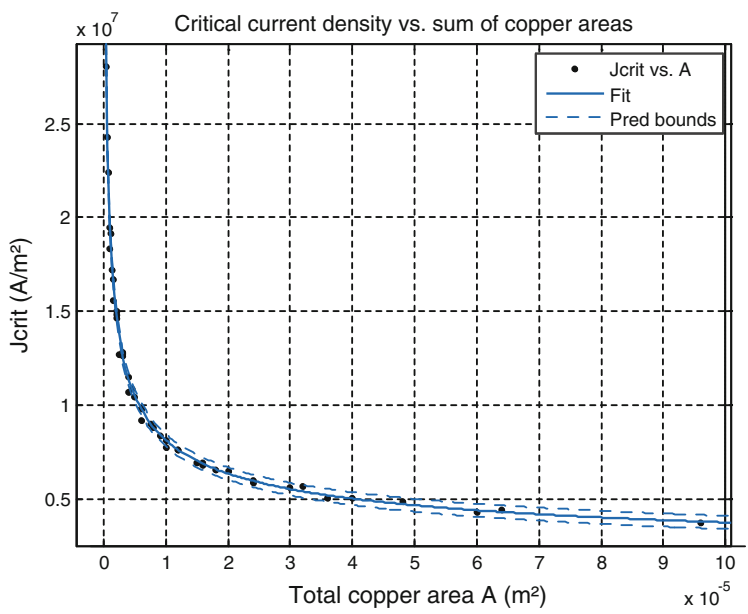

Fig. 6 Polynomial fit for minimum area versus total current of bundle, with prediction bounds

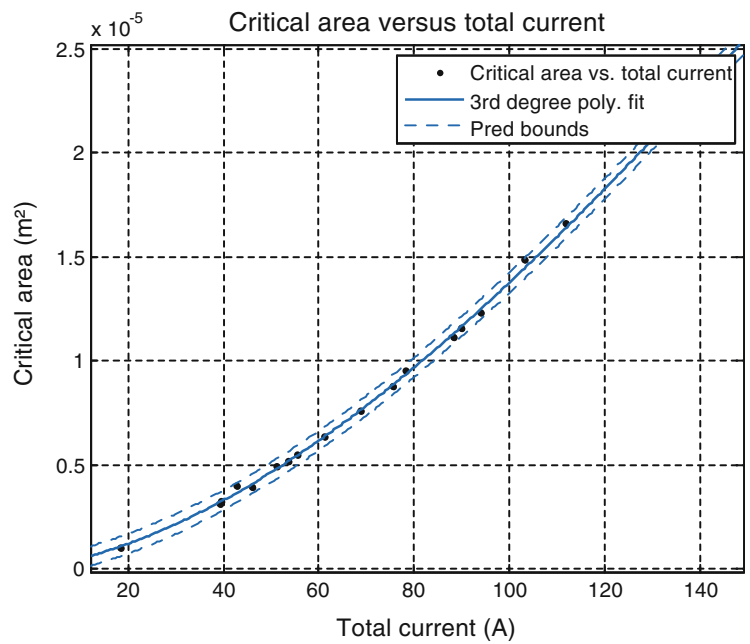

into a simple discrete optimization problem: minimize the area of copper always remaining above its minimum value. This problem will be discussed and solved later in this document.

\section{Regression Models for Wire Bundle Dimensioning}

Linear regressions are carried out with the aim of obtaining general expressions for the critical area of arbitrary wire bundles. With 171 simulated cases and using the stepwise method, we obtained expressions for the logarithms of the critical area and the critical current. 
Fig. 7 Regression model: minimum area of the bundle versus its sum of currents

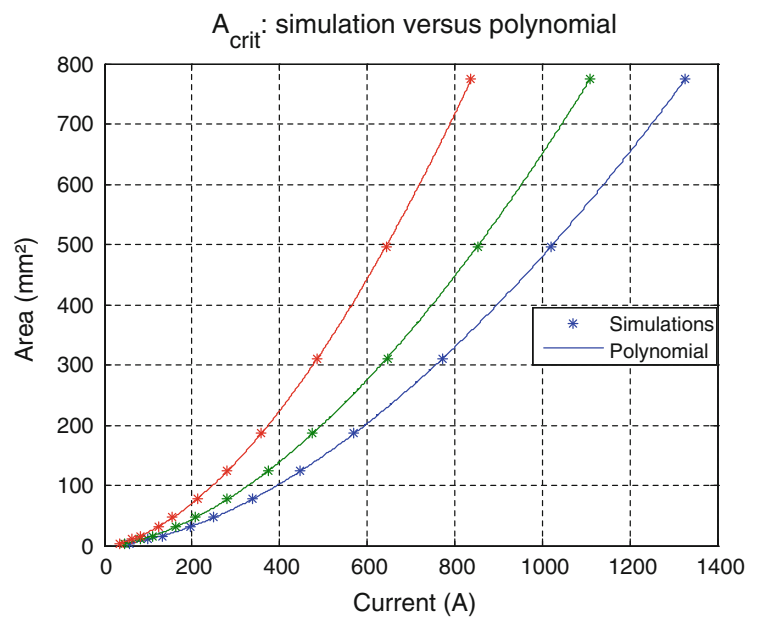

Since what we want to predict in the first case is the critical area, the predictors are reduced to the number of wires $n$, the sum of their currents, $I$ and the ambient temperature $T_{a}$. Moreover, the ratio between copper and PVC can be used as an additional predictor for the response variable $I$. These predictors are included in the regression with pertinent operations such as square root or logarithm to coincide better with the theoretical formulae for cylindrical conductors discussed in this document.

Both regressions have obtained satisfactory results with $R^{2} \approx 1$, especially the one for the critical current, due to the extra predictor related to the mass of PVC versus the mass of copper present in the bundle. This can be observed in Figs. 7 and 8 .

Fig. 8 Regression model: maximum current of the bundle versus its sum of cross-sections

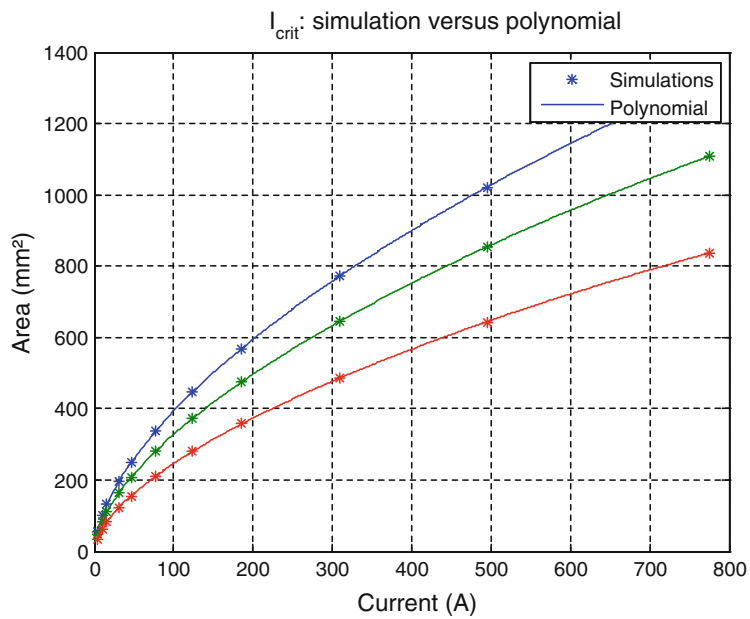




\section{Integer Optimization for Cross-Section Assignment}

As previously discussed, once the critical area is obtained, the cross-sections of the wires must be chosen so that the final area is minimum and greater than its critical value. If it were possible to buy wires with unconstrained cross-sections-i.e. continuous and infinite availability of sizes - then the problem would be easily solved by multiplying each current by the obtained value of current density $J$, obtained by dividing the total current of the bundle by the sum of the areas of the wires $(J=I / A)$. However, cross-sections can be only discrete values, and it is not completely trivial to assign them in an optimum way.

\subsection{Integer Linear Programming}

Let $N$ be the number of wires of the bundle, indexed by $k=1 \ldots N$. Let $Q_{k}$ be a set of available cross-sections for the wire $k$ with $S$ different values of area $a_{k i}, i=1,2 \ldots S$ :

$$
Q_{k}=\left[a_{k 1}, a_{k 2}, \ldots, a_{k S}\right]
$$

Given $N$ wires in a bundle, for each wire there will be $S$ Boolean variables $b_{k i}$ determining whether the wire $k$ is associated to the cross-section $i$ in $Q_{k}$.

$$
x=\left[\begin{array}{c}
b_{11} \\
\vdots \\
b_{1 S} \\
b_{21} \\
\vdots \\
b_{2 S} \\
\vdots \\
\vdots \\
b_{N S}
\end{array}\right] x_{\min }=\left[\begin{array}{c}
0 \\
\vdots \\
0
\end{array}\right]
$$

Since these variables are Booleans, it must be imposed as constraints that they are integers with lower and upper values respectively 0 and 1 . Additionally, since one wire can only have one cross-section, it must be imposed that only one variable for the wire $k, b_{k i}, i=1 \ldots S$ can be 1 , and the rest must be 0 . Specifically, this is achieved by imposing these Booleans to sum exactly 1 :

$$
\sum_{i=1}^{S} b_{k i}=1 \quad k=1, \ldots, N
$$


Expressed in a matrix with $N$ rows and $S \cdot N$ columns:

$$
\begin{gathered}
A_{e q} \cdot x=b_{e q} \\
{\left[\begin{array}{ccc}
11 \ldots 1 & \ldots & 0 \\
\vdots & 11 \ldots 1 & \vdots \\
0 & \ldots & 11 \ldots 1
\end{array}\right] \cdot x=\left[\begin{array}{c}
1 \\
\vdots \\
1
\end{array}\right]}
\end{gathered}
$$

The main constraint of the problem is that the sum of all of the areas must not exceed the given critical or maximum area.

$$
\sum_{k=1}^{N} \sum_{i=1}^{S} b_{k i} \cdot a_{i} \geq A_{\text {crit }}
$$

This can be expressed in matrix form as follows:

$$
\begin{gathered}
A=\left[\begin{array}{lll}
Q_{1} & \ldots & Q_{k}
\end{array}\right] \\
A \cdot x \geq A_{\text {crit }}
\end{gathered}
$$

The objective function of the problem is the total mass of copper, which is not merely proportional to the sum of cross-section areas of the bundle. Instead, we must minimize the volume of copper, for which we need to multiply all of the areas by the length of each wire, $l_{k}$. Additionally, we can multiply each of these volumes $k$ by a relative weight expressing the importance of the wire $k$ in the system. Typically, these relative weights $w_{k}$ will express the expected ratio of manufactured instances of the wire $k$ over the total number of manufactured cars, which is a useful parameter in modular systems. In other terms, we express the volume of copper as the overall sold copper including all of the vehicles of a certain model, rather than the volume of copper of just one car.

$$
V=\left[\begin{array}{lll}
Q_{1} \cdot l_{1} \cdot w_{1} & \ldots & Q_{k} \cdot l_{k} \cdot w_{k}
\end{array}\right] \cdot x
$$

The problem is therefore defined as follows:

$$
\min V\left\{\begin{array}{c}
A \cdot x \geq A_{\text {crit }} \\
A_{e q} \cdot x=b_{e q} \\
0 \leq x \leq 1
\end{array}\right.
$$

Particularly, this method is implemented by giving each wire two possible cross-sections: one with the maximum current density below the critical value, and the other with the minimum current density above the critical value. This defines $Q_{k}=\left[\begin{array}{ll}a_{k 1} & a_{k 2}\end{array}\right]$. Then the algorithm chooses the cross-section for each wire so that the $V$ parameter of the set is minimized, keeping the total area greater than $A_{\text {crit }}$. 
Table 1 Results of optimization of random bundles

\begin{tabular}{l|r|r|r|r|l}
\hline$T_{\text {amb }}\left({ }^{\circ} \mathrm{C}\right)$ & \multicolumn{1}{|c|}{$N$} & $\Sigma I(\mathrm{~A})$ & $A_{\text {crit }}\left(\mathrm{mm}^{2}\right)$ & $A_{\text {total }}\left(\mathrm{mm}^{2}\right)$ & $T_{\max }\left({ }^{\circ} \mathrm{C}\right)$ \\
\hline 62.80 & 8 & 114.99 & 15.63 & 16.00 & 105.17 \\
\hline 79.00 & 24 & 292.78 & 103.62 & 105.00 & 105.26 \\
\hline 67.94 & 12 & 130.87 & 23.45 & 24.00 & 101.47 \\
\hline 82.32 & 25 & 322.06 & 136.15 & 137.00 & 105.72 \\
\hline 60.46 & 27 & 351.90 & 88.10 & 89.00 & 106.55 \\
\hline 62.91 & 29 & 353.80 & 94.37 & 95.50 & 105.75 \\
\hline 59.37 & 18 & 206.56 & 49.36 & 50.50 & 91.66 \\
\hline 59.08 & 5 & 70.03 & 6.28 & 7.25 & 102.12 \\
\hline 81.08 & 11 & 152.34 & 43.36 & 44.00 & 103.56 \\
\hline 72.39 & 34 & 413.26 & 161.46 & 162.50 & 103.60 \\
\hline 71.50 & 8 & 100.22 & 15.33 & 15.50 & 103.89 \\
\hline 59.35 & 31 & 354.96 & 89.88 & 90.00 & 106.13 \\
\hline 80.59 & 21 & 276.46 & 99.52 & 101.00 & 105.02 \\
\hline 73.66 & 37 & 453.54 & 203.45 & 205.00 & 101.25 \\
\hline 65.53 & 5 & 53.08 & 4.53 & 5.00 & 101.66 \\
\hline 70.40 & 18 & 242.48 & 82.53 & 84.50 & 93.57 \\
\hline 67.05 & 6 & 84.28 & 10.08 & 11.00 & 102.74 \\
\hline 57.28 & 36 & 438.63 & 130.62 & 132.50 & 102.74 \\
\hline 62.20 & 3 & 41.73 & 2.87 & 3.00 & 105.91 \\
\hline 58.70 & 30 & 397.17 & 106.47 & 107.00 & 103.26 \\
\hline 60.52 & 31 & 407.05 & 115.65 & 117.00 & 103.31 \\
\hline 62.20 & 33 & 428.68 & 132.92 & 133.00 & 104.19 \\
\hline 67.52 & 5 & 35.60 & 2.46 & 2.50 & 103.78 \\
\hline & & & & & \\
\hline
\end{tabular}

The optimization algorithms assumes that the critical area obtained by means of the regression model provides a bundle with a maximum temperature of $T_{\max }$, in this case $T_{\max }=105{ }^{\circ} \mathrm{C}$. In order to validate the system, some simulations of random bundles have been performed (see Table 1).

\section{Conclusions}

We obtained expressions that provide the minimum sum of cross-sections of a wire bundle with given currents and algorithms capable of assigning feasible cross-sections to them. Results show great accordance between the models and the finite-element simulations for both the expressions of minimal area for given currents and maximum current for given area. This satisfactory result ought to be now complemented with experimentation, which is expected to be in accordance to the obtained results due to the already good accordance of the finite elements simulations and reality. 
As for the cross-section assignment algorithm, the linear method provides the best results regarding maximum temperature reached in the bundle, which is rarely above $105{ }^{\circ} \mathrm{C}$. It is now necessary to validate the result of these expressions and dimensioning algorithms experimentally in order to find imperfections in the actual maximum temperature of the bundles.

\section{Future Work}

Actual wire harnesses include in many cases mixed types of wires, with different materials. That is why throughout the elaboration of this study it has come to our minds the necessity of including the presence of different materials to the bundles. It seems crucial to extend this study finding regression models that include the proportion of different typical materials in the bundle, such as other isolation materials or aluminium.

In a similar way, the purpose of optimizing the used mass of copper in the wiring harnesses, it appears useful to consider how can these so-called cold signal wires inside the bundle influence the maximum temperature. It could be done by including in these further regression models not only the ratio of different materials but also information about wires with negligible currents. It is expected that the presence of these wires will provide more favourable results, in such a way that some copper could be saved. Nevertheless, this must be validated with results.

\section{References}

1. Ilgevičius, A.: Analytical and numerical analysis and simulation of heat transfer in electrical conductors and fuses. Universität der Bundeswehr München (2004)

2. Čiegis, R., Ilgevičius, A., Ließ, H., Meilūnas, M., Suboč, O.: Numerical simulation of the heat conduction in the electrical wires. Math Model Anal 12(4), 425-439 (2007)

3. Frei, S., Diebig, M.: Simulation-based optimization of multi voltage automotive power supply systems. In: Vehicle Power and Propulsion Conference (VPPC), IEEE, pp. 1-6. 15-18 Oct 2013

4. Diebig, M., Frei, S.: Modeling of the automotive power supply network with VHDL-AMS. In: Vehicle power and propulsion conference (VPPC), IEEE, pp. 1-3 (2010)

5. Čiegis, R., Meilūnas, M., Jankevičiūtè, G., Ilgevičius, A.: Determination of heat conductivity coefficient of a wire bundle by inverse problem solution method. Electron. Electr. Eng. ISSN 1392-1215. 2(90) (2009)

6. Loos, F., Dvorsky, K., Ließ, H.: Two approaches for heat transfer simulation of current carrying multicables. Math Comp. Simul. 101, 13-30 (2014)

7. Benthem, R.C. van, Grave, W. de, Doctor, F., Nuyten, K., Taylor, S., Jacques, P.A.: Thermal analysis of wiring for weight reduction and improved safety. National Aerospace Laboratory NLR. NLR-TP. AIAA/ICES Conference, Portland, Oregan, USA 18-21 July 2011

8. Harbrecht, H., Loos, F.: Optimization of current carrying multicables. Comput. Optim. Appl. (2013) 
9. Ließ, H.: Optimal dimensions for electrical conductors in mobile systems. In: Electric drives production conference (EDPC), $1^{\text {st }}$ international. IEEE, pp. 139-142 (2011)

10. Olsen, R.S., Holboll, J., Gudmundsdóttir, U.S.: Dynamic temperature estimation and real time emergency rating of transmission wires. In: Power and energy society general meeting IEEE, pp. 1-8, 22-26 July 2012

11. Loos, F., Ließ, H., Dvorsky, K.: Simulation methods for heat transfer processes in mechanical and electrical connections. In: Electric drives production conference (EDPC), $1^{\text {st }}$ international. IEEE (2011)

12. Short, T.A.: Electric power distribution handbook. CRC Press LLC, USA (2004)

13. Lewis, R. W., Nithiarasu, P., Seetharamu, K. N.: Fundamentals of the finite element method for heat an fluid flow. Wiley, New York (2004)

14. Blomberg, T.: Heat conduction in two and three dimensions: computer modelling of building physics applications. Lund University, Sweden (1996)

15. VDI-Heat atlas, 2nd edn. Springer, Berlin (2010)

16. Hussain, K.M., Hussain, D.: Telecommunications and networks. Butterworth-Heinemann, UK (1997)

17. Brabetz, L., Ayeb, M., Jilwan, G., Gräbel, P., et al.: A new approach to the test, assessment and optimization of robust electrical distribution systems. SAE Int. J. Mat. Manuf. (2013)

18. Combettes, S., Sontheimer, T., Rougemaille, S., Glize, P.: Weight optimization of aircraft harnesses. In: Advances on practical applications of agents and multi-agent systems. Advances in intelligent and soft computing, vol. 155, pp. 229-232 (2012)

19. Lin, C., Rao, L., D'Ambrosio, J., Sangiovanni-Vincentelli, A.: Electrical architecture optimization and selection - cost minimization via wire routing and wire sizing. SAE Int. J. Passeng. Cars-Electron. Electr. Syst. (2014) 